\title{
EL PRINCIPIO DE LA INTEGRALIDAD
}

\author{
Rubén Darío Lésmes Bustamante ${ }^{\dagger}$ \\ rlesmes3@gmail.com \\ ${ }^{\dagger}$ Piloto - Instructor de Vuelo - Consultor de la Industria Aeronáutica
}

14 de octubre de 2020

\begin{abstract}
Resumen
Se ha determinado la necesidad de la inclusión articulada de las competencias actitudinales como pate esencial en la selección, formación, evaluación y promoción del personal aeronáutico. La gestión de la seguridad aeronáutica ha evolucionado en tres aspectos fundamentales: Las habilidades técnicas, las habilidades cognitivas y las habilidades actitudinales, las competencias actitudinales y los comportamientos de los individuos han demostrado sin lugar a duda un éxito en la disminución del riesgo operacional.

Se ha hecho un estudio transversal de las estadísticas de la accidentalidad tanto de las aerolíneas como de la aviación general y, la evolución del pensamiento en materia de la gestión de la seguridad demostrando que el comportamiento humano afecta directamente los índices del riesgo. Con base en el estudio de las estadísticas y de los modelos de gestión vigentes se ha propuesto El principio de la Integralidad como el fundamento de actualización de los programas de selección, formación, evaluación y promoción del personal que se desempeña en la industria aeronáutica, proponemos el modelo integral como la inclusión definitiva y articulada de las competencias no técnicas o blandas en el recurso humano.

El integrar articuladamente a las competencias técnicas, cognitivas y actitudinales disminuye la probabilidad y la severidad de los eventos en materia de la seguridad. El principio de la integralidad va dirigido a las organizaciones de la industria aeronáutica (ATC, Autoridades aeronáuticas, centros de instrucción, fabricantes, aerolíneas, operadores, talleres, centros de despacho, a los profesionales en factores humanos) y en general a todos quienes participan de manera sistémica en el desarrollo y ejecución de la actividad aérea.
\end{abstract}

Palabras Clave: Aviación, Psicología Aeronáutica, Integralidad. 


\section{Índice}

1. Introducción $\quad 3$

1.1. El componente humano . . . . . . . . . . . . . . . . . . . 4

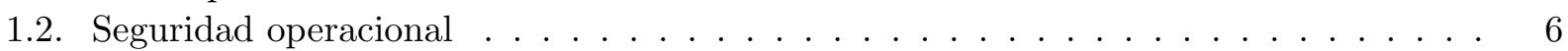

1.3. Herramientas especiales de gestión del riesgo . . . . . . . . . . . . . . . 7

1.4. La empresa organizacional . . . . . . . . . . . . . . . . . . 8

1.5. Modelos de análisis de accidentes . . . . . . . . . . . . . . . 9

1.5.1. Modelo secuencial . . . . . . . . . . . . . . . . . . . . 9 9

1.5.2. Modelo epidemiológico . . . . . . . . . . . . . . . . . . 10

1.5.3. Modelo sistémico . . . . . . . . . . . . . . . . . . . . . 11

2. Planteamiento de la problemática $\quad 11$

2.1. Causas probables de la problemática . . . . . . . . . . . . . . . 12

2.1.1. Participación de las autoridades aeronáuticas de los estados . . . . . . . 12

2.1.2. Formación de personal aeronáutico . . . . . . . . . . . . . . . . . . 13

2.1.3. Profesionales de la salud y factores humanos en la industria aeronáutica . . . 13

2.2. Organizaciones aeronáuticas . . . . . . . . . . . . . . . 14

3. El principio de la integralidad $\quad 14$

3.1. El componente actitudinal . . . . . . . . . . . . . . . . . 15

3.2. Implementación del principio de la integralidad . . . . . . . . . . . . . 15

4. Conclusiones 16 


\section{Introducción}

La industria aeronáutica ha sido considerada como la más segura en referencia a los índices de la accidentalidad, no obstante, llegar a éstos índices no es el resultado de avances coincidenciales, más bien, ha sido el resultado del estudio y la gestión en materia del componente técnico, humano y organizacional dentro del marco de las estrategias reactivas de gestión, aunque siempre en la búsqueda de la proactividad y la predictividad de los eventos de seguridad.

El pensamiento en materia de la seguridad ha evolucionado desde el génesis de la industria en los años mil ochocientos con la aparición de los primeros aerostatos hasta la actualidad con los fundamentos de la seguridad operacional y organizacional, por lo tanto, las estrategias de gestión del riesgo han sufrido cambios significativos avanzando paralelamente con las eras de la industria, éstas estrategias se pueden clasificar en tres aspectos: Estrategias de desarrollo tecnológico, estrategias de desarrollo y entendimiento del factor humano y estrategias de la implementación de la seguridad operacional que termina siendo una seguridad organizacional. (Figura 1)

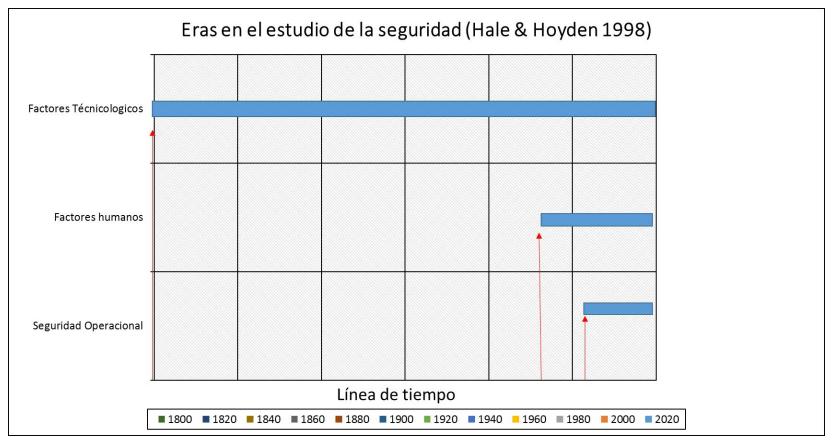

Figura 1: Eras en el estudio de la seguridad (Haley \& Hayden 1998)

Al hacer un análisis de la casuística de la accidentalidad desde los años 40's podemos ver cómo la tendencia en los índices marca una disminución significativa en los últimos años.

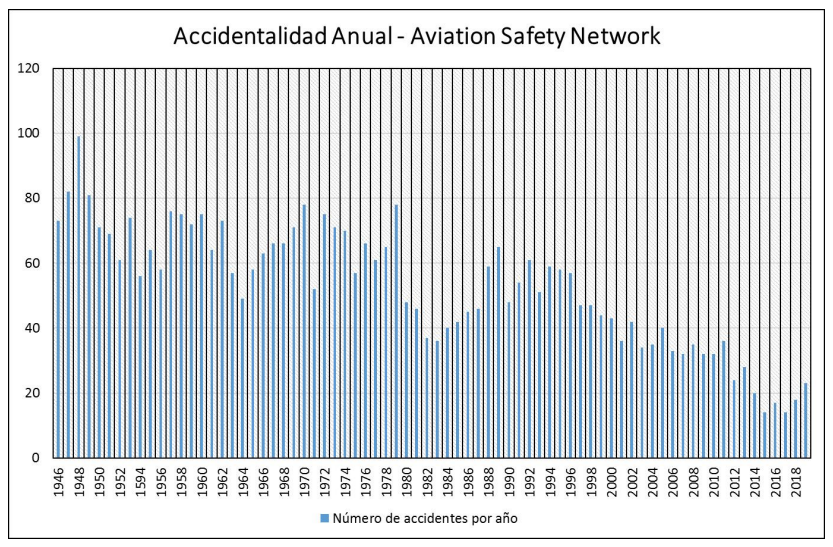

Figura 2: Número de accidentes por año (Aviation Safety Network)

Si hacemos un análisis en paralelo de las líneas de tiempo en materia de las estrategias de gestión y la casuística de la accidentalidad concluiremos que la carrera tecnológica, aunque es de 
suma relevancia no es el todo en la obtención de los resultados en materia de la seguridad, es así como a partir de los años 40's empieza a verse una disminución en la curva de la accidentalidad, pero si observamos con detenimiento, es realmente después de los años 90's cuando en definitiva los índices de la accidentalidad reflejan una tendencia de disminución constante.

La industria aeronáutica ha aportado más de doscientos años en la evolución tecnológica y es evidente que a partir del nacimiento de la presurización, automatización, sistemas de alerta y control de tránsito, sistemas de navegación de alta precisión, etc. Se ha logrado mitigar la probabilidad de los eventos en la operación aérea, no obstante a lo anterior, la industria ha trabajado sólo los últimos cincuenta años en el entendimiento y mejoramiento de los factores humanos y es allí en donde la gestión no solo demostró una reducción en la probabilidad sino que también en la severidad de los eventos, por ello hablamos del riesgo como la probabilidad y severidad de los eventos, ahora bien, la industria ha propuesto sólo en los últimos treinta años la inclusión del concepto "Cultura de la seguridad operacional" como una estrategia clave para la mitigación del riesgo, esto en la actualidad ha llevado a los índices de la accidentalidad a su media más baja considerando además que el crecimiento operacional ha sido exponencial y global en las últimas décadas.

\subsection{El componente humano}

De la misma manera en que las eras de la gestión han evolucionado en referencia a las estrategias de gestión de la seguridad; el perfil, el enfoque de la evaluación y valoración de los individuos que se desempeñan en la industria aeronáutica ha evolucionado considerablemente. (Figura 3)

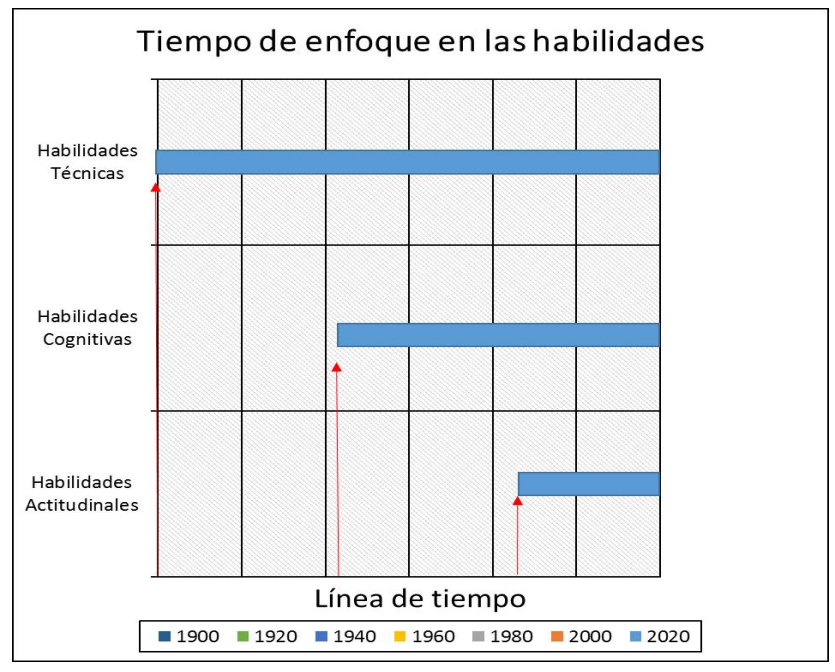

Figura 3: Tiempo de enfoque en las habilidades

La evolución del enfoque se da en razón a las necesidades de la industria, es así como en sus orígenes y como consecuencia de la incipiente tecnología se requería no solo del arrojo de las personas que pretendían volar (nótese que se habla básicamente del piloto), sino que también de unas destrezas enfocadas en las habilidades técnicas. 
En sus orígenes, la industria no contaba con tecnologías en lo referente a la navegación aérea, control de tránsito, materiales y sistemas que protegieran al individuo de la composición física y química de la atmósfera, ambiente en el que por naturaleza el ser humano es frágil. Se requirió por supuesto de hombres con habilidades mecánicas en referencia a la coordinación, orientación y control, el piloto en definitiva debía ser una persona bastante hábil en lo técnico.

Con el nacimiento de los sistemas autónomos de control de vuelo, control de tránsito, sistemas de advertencia, mediciones del ambiente atmosférico y otras tecnologías, se dio paso a la segunda generación de los individuos de la industria y es la generación del conocimiento, el piloto y ahora también el técnico de mantenimiento (nótese como se amplía el espectro de incidencia en la necesidad de los perfiles del ser humano), debían demostrar capacidades de entendimiento y comprensión de los sistemas y tecnologías nuevas, así como también la capacidad de operarlos, en este sentido las habilidades técnicas básicas de coordinación, orientación y control (aunque no son necesariamente excluidas en su contexto), cedieron ante las habilidades cognitivas.

El esfuerzo de la industria en la mejora del aspecto tecnológico y el desarrollo de las habilidades técnicas y cognitivas trajo consigo una reducción significativa de estos factores como causa raíz de la accidentalidad, esta reducción muestra a partir de los años 70's un aparentemente nuevo factor causa a todo nivel en la accidentalidad global, el factor humano. (Figura 4)

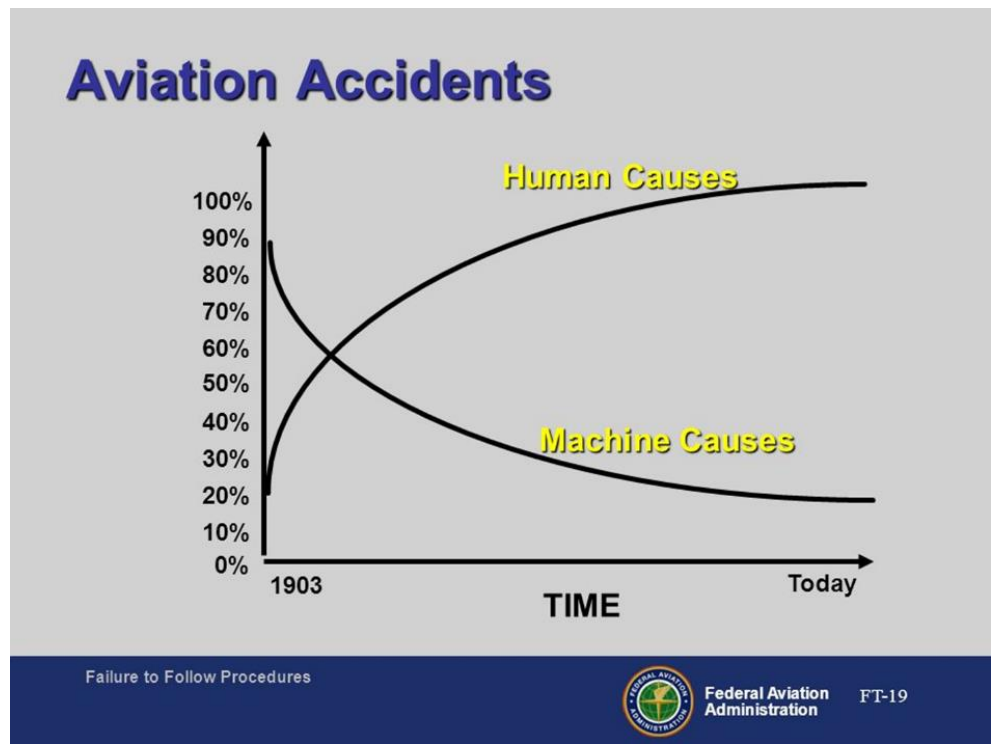

Figura 4: Diapositiva de Referencia: "Failure to follow procedures FAA"

Desde los años 90's y hasta la actualidad la industria ha demostrado que es imprescindible entender a las organizaciones aeronáuticas de manera sistémica, entendiendo como sistema a cualquier entidad consistente en un conjunto de elementos interrelacionados y en interacción que están organizados para realizar una función, o cumplir un objetivo común, la industria aeronáutica es sin lugar a duda un conjunto de subsistemas (organizaciones) interrelacionados unos con otros. Desde la perspectiva sistémica de la industria aeronáutica, el individuo que se desempeña en ella debe comprender que hace parte de un sistema y que su desempeño afecta directa e indirectamente 
los resultados de las diferentes organizaciones de la industria, es allí en dónde el comportamiento como parte del todo se hace relevante, nace entonces el enfoque de las actitudes y la necesidad de evaluar las destrezas no técnicas (cooperación, liderazgo y gestión, conciencia situacional y toma de decisiones).

Nuevamente, si hacemos un análisis transversal de la casuística entre las relaciones: Evolución del pensamiento en materia de la seguridad - Índices de la accidentalidad - Enfoque de las habilidades, veremos claramente que la disminución progresiva de la accidentalidad se debe en razón a la implementación de la cultura de la seguridad operacional y a las habilidades actitudinales o no técnicas de los individuos de las organizaciones aeronáuticas (nótese como el espectro de influencia en la gestión ahora incluye a todo el personal que hace parte del sistema).

\subsection{Seguridad operacional}

La seguridad operacional se ha definido como un estado, "Es el estado en el que el riesgo de lesiones a las personas o daños a los bienes se reduce y/o se mantiene en un nivel aceptable mediante un proceso continuo de identificación de peligros y gestión del riesgo", éste estado o condición, como la homeóstasis, hace referencia a un equilibrio, en la industria aeronáutica el equilibrio se da entre la operación y el riesgo, es decir la operación (y con ella la productividad) puede crecer siempre y cuando se gestione el riesgo que conlleva ése crecimiento (más operación significa más riesgo), por lo contrario crecer e incluso operar sin la gestión del riesgo, llevará sin duda a las organizaciones a una exposición directa y, como consecuencia, a sufrir eventos de seguridad sean ellos situaciones no deseadas, incidentes o accidentes.

No es coincidencia que la industria haya determinado que la necesidad primaria de la seguridad operacional sea La cultura, ya que en definitiva ningún emprendimiento está libre de afectaciones culturales, así entonces, si se debe comprender a la industria aeronáutica de manera sistémica en dónde los individuos hacen parte de conjuntos que confluyen en un mismo objetivo es imprescindible alinear los aspectos culturales que son tan diversos como la población misma de la industria (existen entre otras: la cultura generacional, geográfica, organizacional, de género, etc.) por lo tanto, establecer los lineamientos de la cultura de la seguridad operacional y promoverlos en la industria requiere de un sistema de gestión que garantice su adherencia, esto es el Safety Management System, o SMS.

Los lineamientos de la cultura de la seguridad operacional se encuentran dentro el marco de los sistemas de aseguramiento de la calidad, ellos son: Entrenamiento, procesos, medición, documentación y evaluación, esto quiere decir que los individuos que pretenden desempeñarse en la industria aeronáutica de hoy, deben demostrar habilidades no técnicas con el fin de asimilar y/o adherir el perfil que le permita trabajar en un entorno que: documenta, mide, entrena, evalúa y gestiona procesos, vuelve en éste punto a ser relevante el componente actitudinal a todo nivel de las organizaciones ya que es el comportamiento del individuo el que hace la diferencia en los resultados de la operación y en la afectación del sistema. 


\subsection{Herramientas especiales de gestión del riesgo}

Asegurar la implementación de la seguridad operacional mediante el SMS exige comprender lo que contiene un sistema de gestión y el componente que logra el cambio.

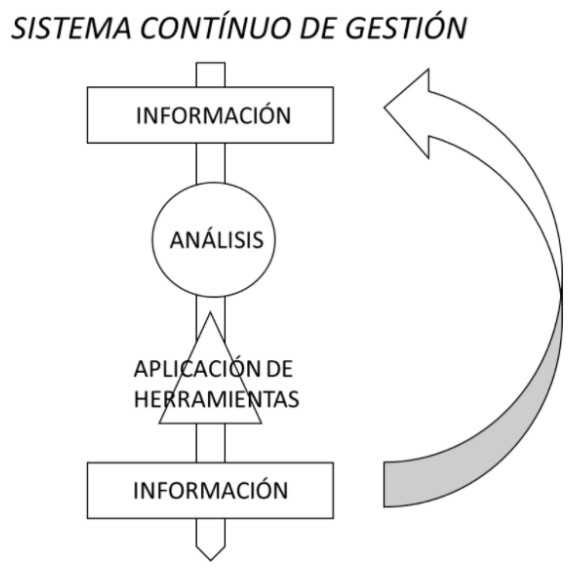

Figura 5: Sistema continuo de gestión

En el sistema continuo de gestión (Figura 5), la organización recolecta información proveniente de las operaciones, la información es depurada y analizada con el fin de contrastarla contra los índices de tolerancia de la organización (en este caso en particular hablamos de los índices de tolerancia del riesgo) de tal manera que según sea la tolerancia, se propondrán las defensas y herramientas de gestión que han sido diseñadas para la mitigación del riesgo, una vez aplicadas las defensas y herramientas de gestión, se deberá medir el sistema con el fin de obtener la información residual.

Si la información residual se analiza dentro del sistema nuevamente, tenemos el funcionamiento de un sistema continuo de gestión que opera hasta lograr los niveles más bajos posibles en la probabilidad y severidad de las consecuencias de los peligros identificados.

Son por supuesto las defensas y herramientas de gestión del riesgo las que logran el cambio dentro de las organizaciones, en el marco de la cultura de la seguridad operacional se consideran tres defensas: El entrenamiento, los reglamentos y las tecnologías, no obstante, aunque parece un intangible se debe considerar a la supervisión como una herramienta esencial y contemplada dentro de los modelos de gestión de la seguridad.

Las herramientas de gestión del riesgo por su parte han evolucionado desde los años 70's y han surgido como consecuencia de la investigación de los accidentes y el estudio de la casuística, podemos incluir entre ellas: Modelo TEM (Threat and Error Management), ALARP (Approach and Landing Accident Reduction Program), CFIT (Controlled Flight in to Terrain), CRM (Company Resource Management), etc.

Cuando se estudian las herramientas especiales de gestión de la seguridad operacional se deduce con facilidad que todas y cada una de ellas se centran en el factor humano y en las competencias no 
técnicas con el fin de obtener resultados directos en la operación, el modelo SHELL -C, el modelo TEM, son un claro ejemplo de ello en donde se identifica al comportamiento del individuo como el factor determinante en el resultado de la operación, en profundidad la asimilación de las buenas prácticas y las competencias no técnicas tienen inherencia directa en la búsqueda de la condición segura cuando aparecen condiciones no deseadas, errores, amenazas, y otros fenómenos. Nuevamente el componente actitudinal es pieza clave en la gestión del riesgo.

\subsection{La empresa organizacional}

De acuerdo a Bolman \& Deal 1984, el comportamiento organizacional se da en cuatro marcos: Marco Estructural, Marco de los Recursos Humanos, Marco Político, Marco Simbólico/Cultural, éstos marcos por supuesto denotan la existencia de una cultura organizacional que incluye en ella los ambientes y climas organizacionales dados por Westrum 1994; si se considera a la organización como parte del sistema, se debe considerar que las organizaciones y sus culturas afectan directamente el resultado de las demás organizaciones que tienen el fin común de la industria aeronáutica que en todo caso será mantener tan bajos como sea razonable a los índices de la seguridad operacional, en éste sentido la industria ha propuesto el concepto de "La empresa organizacional", con el ánimo de establecer una estructura que si bien no es un organigrama, contiene los aspectos claves de gestión a nivel de las organizaciones. (Figura 6)

\section{EMPRESA ORGANIZACIONAL}

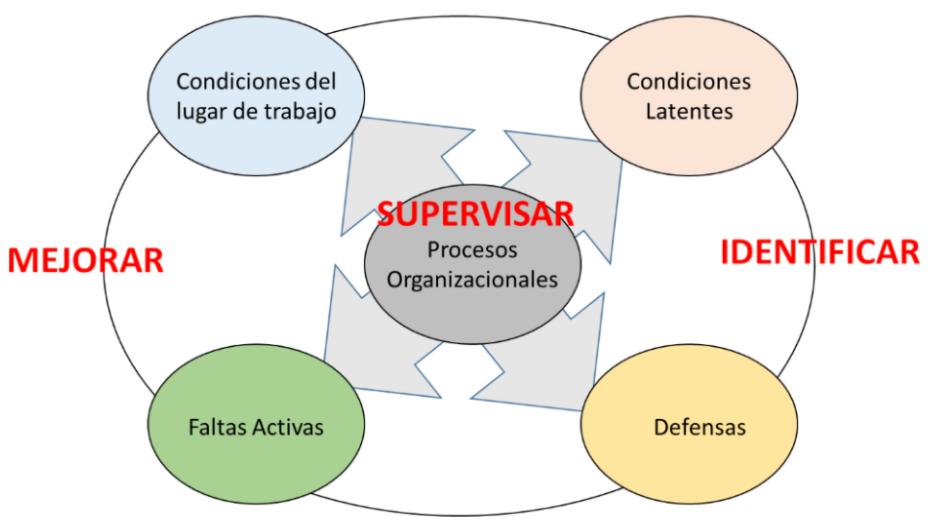

Figura 6: La empresa organizacional

Bajo los preceptos de la empresa organizacional (Ver gráfica 6.0), la organización como parte de un sistema buscará mejorar las condiciones del lugar de trabajo de los empleados y la promoción de una cultura justa en el capítulo de los errores y violaciones, de otra parte, la organización identificará bajo el principio de la causalidad todos aquellos aspectos que denoten peligros y/o riesgos para la operación, así mismo, propenderá por la identificación de las defensas y el mejoramiento de las mismas.

En el cabal cumplimiento de este objetivo la organización diseñara y trabajara dentro del marco de los procesos organizacionales anexando a los procedimientos existentes (operativos, técnicos, administrativos y financieros), los componentes de alcance, evaluación, norma técnica, documentación 
y mejora continua.

El compromiso del alto ejecutivo para con el cumplimiento de esta implementación exige la asimilación de las competencias no técnicas y/o, operacionales, así como el estudio de los factores humanos con el fin de promover los valores y fundamentos de la cultura de la seguridad operacional a todos los niveles de la organización, es así como las organizaciones de hoy se acompañan asertivamente de los profesionales del área en referencia a los factores humanos fisiología y psicología aeronáutica, al accidente que tiene como causa raíz la falla en uno de los componentes de la empresa organizacional se le conoce como el "accidente organizacional".

\subsection{Modelos de análisis de accidentes}

La investigación de accidentes en la industria aeronáutica ha sido desarrollada mediante el uso de modelos, estos ayudan a identificar los factores determinantes y las causas probables de los eventos, éstos modelos utilizados han evolucionado en el tiempo y en la comprensión del entorno complejo de las organizaciones, se cuentan entre ellos los siguientes:

\subsubsection{Modelo secuencial}

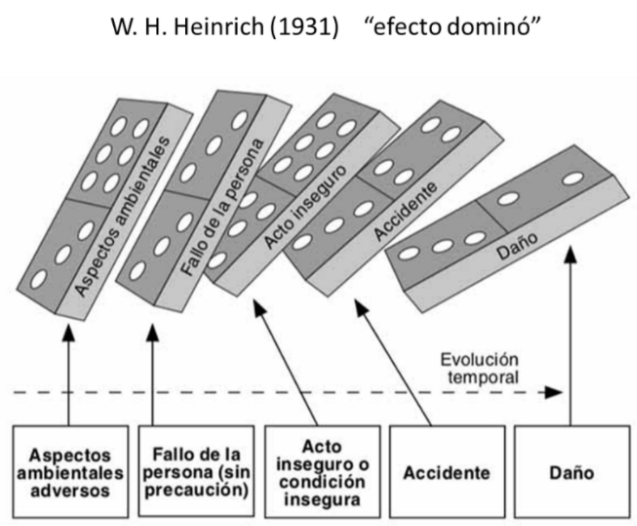

Figura 7: Modelo secuencial de investigación de accidentes (W.H. Heinrich (1931)

En este modelo, el accidente se conceptúa como una sucesión de causas y efectos que suceden de manera secuencial o lineal en un determinado orden, por ser lineal simple sus componentes se pueden descomponer de manera sencilla, lo que significa que identificando los componentes se puede inferir de manera rápida en las causas de la accidentalidad, de la misma manera la gestión se basa en el hecho de evitar componentes identificados, lo que en un domino sería retirar una ficha para romper la inercia de los eventos. Éste modelo se centra en la investigación de los hechos en la operación misma, función que fue el deber ser de la seguridad aérea. 


\subsubsection{Modelo epidemiológico}

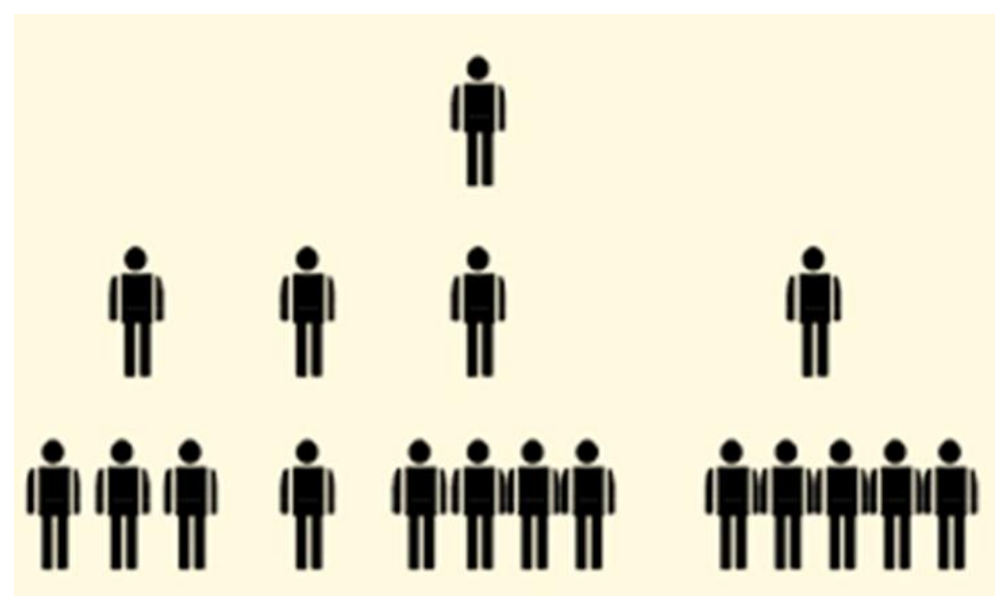

Figura 8: Modelo Epidemiológico de investigación de accidentes

Los Modelos Epidemiológicos son también llamados organizacionales, éstos modelos pueden ser vistos como una extensión de los modelos secuenciales ya que se descomponen de manera lineal aunque no de una manera simple ya que éste va más allá de lo inmediato, su espectro se amplía más allá de los límites de la operación misma involucrando factores y causas latentes que no son percibidos de manera directa sino más bien que se evidencian por factores desencadenantes, un ejemplo de las condiciones latentes es la deriva práctica que se fundamenta en la desviación de los procedimientos normales en el tiempo, la deriva se torna en riesgo latente hasta que se evidencia por un accidente, bajo éste modelo las situaciones no deseadas y/o los hallazgos no se estudian como la posible causa de un evento sino más bien como la consecuencia de un factor no determinado y que existe previamente en el sistema. En la ocurrencia de un evento de seguridad, el objetivo de la investigación bajo el modelo epidemiológico es identificar el proceso organizacional que ha sido afectado.

Un ejemplo del modelo epidemiológico es el Modelo de Falla Simultánea (J. Reason). (Figura 9)

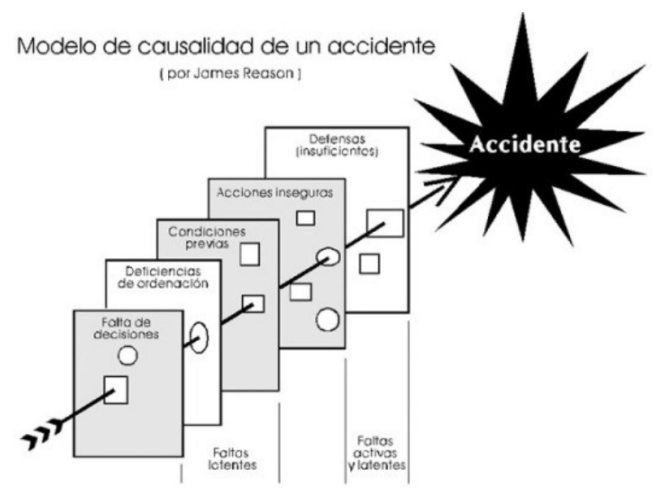

Figura 9: Modelo de la causalidad en la investigación de accidentes (J. Reason) 


\subsubsection{Modelo sistémico}

A diferencia de los modelos lineales que describen el accidente como causa y efecto y que adolecen de la capacidad de entender a la industria aeronáutica de manera sistémica y al accidente de manera holística, el modelo sistémico comprende a la organización como parte de un todo y al accidente como consecuencia de la interrelación de varios sistemas globales y complejos, el modelo sistémico no es lineal, no se descompone.

Durante la investigación de los accidentes bajo el modelo sistémico se entenderá a la organización como una red intercomunicada con eventos y relaciones, las organizaciones son sistemas sociales y técnicos en dónde la dinámica vincula a las personas con la tecnología, con las normas, con el medio ambiente, etc. En la ocurrencia de un accidente el objetivo será determinar ¿qué llevo a las personas al evento? y no, qué regla o proceso se rompió o violó.

Siendo la actividad aeronáutica parte de un modelo sistémico y entendiendo a las organizaciones como parte de un todo, es de suma importancia que el elemento humano que conecta todas las interrelaciones (y que sin duda es el pilar central de la gestión a todo nivel organizacional y de la industria), desarrolle la capacidad de administrar y evaluar el entorno, retornamos ineludiblemente a las competencias no técnicas del individuo que ahora incluyen no solamente a las áreas operativas y técnicas sino que más bien incluyen a todas las organizaciones que confluyen en la actividad aeronáutica (Centros de control, autoridad aeronáutica, despacho, auxiliares de cabina, personal administrativo, fabricantes, profesionales de la salud vinculados a la industria, pilotos, técnicos de mantenimiento, etc.).

\section{Planteamiento de la problemática}

La Organización de Aviación Civil Internacional ha concluido por lo ya antes expuesto que los accidentes en la industria aeronáutica seguirán ocurriendo, la eliminación de los riesgos resulta una tarea improbable y la eliminación del error humano como una acción o inacción involuntaria es imposible, por lo tanto se ha propuesto promover la seguridad operacional como una cultura de gestión que logre mitigar al menos la probabilidad y la severidad de la ocurrencia de los eventos y enmarcarla dentro del aseguramiento a la calidad de sus procesos (SMS Safety Management System y SSP Safety State Program) lo que involucra a la autoridad aeronáutica de cada estado.

A la fecha y luego de treinta años de promoción de la estrategia de gestión, el panorama de asimilación e inclusión de la cultura dentro de las organizaciones es desalentador si consideramos el hecho de manera holística y a la industria aeronáutica como el todo. Esto es debido a que la asimilación de la cultura y la adherencia a los programas se ha logrado de manera significativa solo en un segmento de la aviación, este es el segmento llamado de operación regular o aerolíneas, es allí en donde sin lugar a duda se reflejan los bajos índices de accidentalidad, sin embargo, la asimilación en los otros segmentos como lo son: Aviación de operación no regular o chárter, aviación ejecutiva, aviación corporativa, trabajos aéreos especiales (Fumigación, ambulancias, sísmica, fotografía, etc.) y centros de instrucción, la seguridad operacional se asoma con timidez y con bastante recelo en la capacidad de adherencia de sus individuos, consecuencia de ello se revelan los altos índices de la accidentalidad en estos segmentos de la industria aeronáutica. 
Debemos visualizar de manera objetiva que la aviación regular es apenas la punta del Iceberg de lo que realmente es la industria aeronáutica, lo que no se ve, o no se refleja, es la proporción mayor de lo que realmente es esta industria. (Figura 10)

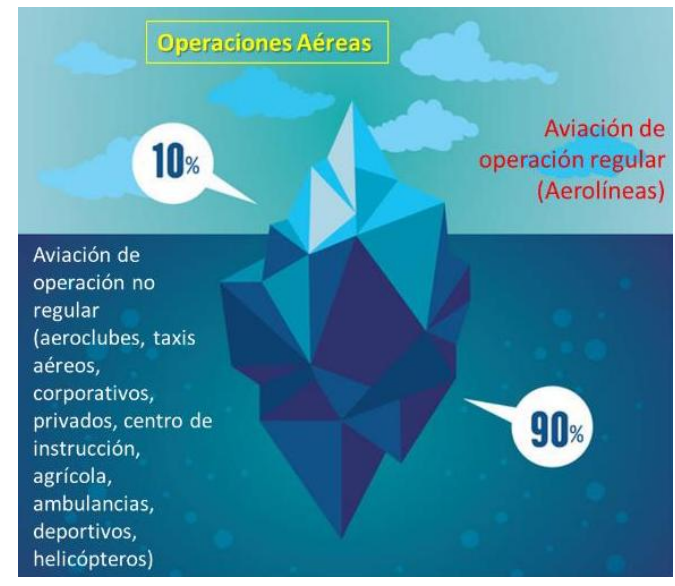

Figura 10: Distribución de la operación aérea en la industria aeronáutica

\subsection{Causas probables de la problemática}

Se pueden identificar varias causas probables sobre la pobre asimilación de las estrategias de gestión, entre ellas podemos mencionar las siguientes:

\subsubsection{Participación de las autoridades aeronáuticas de los estados}

Si bien es cierto que la Organización de Aviación Civil Internacional a través de su anexo 19 y documento 9859 ha determinado las características, el alcance y los pilares de la implementación de los sistemas de gestión del riesgo y ordena su implementación, de manera local, las autoridades aeronáuticas carecen de suficientes recursos humano, técnico y organizacional que promueva de manera asertiva lo ordenado.

Es claro que no basta con informar sobre las necesidades del sistema, la promoción del mismo debe incluir socialización y entrenamiento, para ello, la autoridad aeronáutica debe contar con un personal que contenga la cultura de la seguridad operacional en sí mismo y un alto desempeño en competencias no técnicas que conlleven a la promoción asertiva y a la asimilación por parte de las organizaciones de la industria.

El reglamento no lo es todo, si la autoridad aeronáutica considerada como una parte esencial del sistema, no contiene al equipo humano con las capacidades, habilidades y destrezas actitudinales que exige la cultura de la seguridad operacional, así como a los ejecutivos responsables entrenados de manera adecuada en la concepción de la industria como un sistema interrelacionado, el efecto que tendrá en la promoción será poco satisfactorio.

Es imprescindible que el factor humano que compone la organización de la autoridad aeronáutica adhiera los principios y las destrezas no técnicas, así como a la cultura de la seguridad operacional, 
de esta manera se desarrollará un programa estatal de la seguridad SSP que sea amigable con los sistemas interrelacionados de las organizaciones operadoras y de prestación de servicios aeronáuticos, así como de la industria en general.

\subsubsection{Formación de personal aeronáutico}

Los centros de instrucción aeronáuticos, aeroclubes y otras entidades de formación aún operan bajo el principio de las habilidades técnicas y cognitivas, esto debido en gran parte a que las autoridades locales no han incluido dentro de sus reglamentos a los programas especiales y modelos de gestión de la seguridad operacional más allá del CRM, los programas de entrenamiento con que aún hoy en día se forma al personal de la industria no han sido actualizados considerando las nuevas exigencias actitudinales del personal, esto redunda en la poca competencia de los alumnos egresados y la poca aceptación de las empresas regulares de los mismos, por este motivo algunas compañías fabricantes de aeronaves como Airbus han tomado la decisión de invertir en los centros de formación ya que debido al panorama actual no hay suficientes pilotos con los estándares que requiere la industria, aunque existan cientos de egresados de los centros de instrucción aeronáutica.

Esta condición ha creado un círculo vicioso en donde el egresado que no cumple con el estándar de la industria aeronáutica regular se queda laborando en la industria no regular que no ha sido permeada por esta cultura organizacional, esto conlleva a una baja capacidad de gestión del riesgo por lo que los índices de la accidentalidad se mantienen altos en el segmento de las empresas consideradas de operación no regular.

De otra parte, los entrenamientos recurrentes que deben cumplir con el objetivo de actualizar al personal de la industria e incluir la nueva cultura de la seguridad operacional en cada individuo, se han quedado desactualizados bajo el esquema de entrenamiento en las destrezas técnicas y cognitivas, aumentando con ello la deriva que ya lleva más de treinta años.

\subsubsection{Profesionales de la salud y factores humanos en la industria aeronáutica}

A partir de los años 70's se ha incluido el estudio de los factores humanos en el personal de la industria, esto es, las condiciones fisiológicas y psicológicas del individuo, éstos estudios han evolucionado de manera asertiva evaluando el desempeño de las personas desde la cabina de mando hasta las organizaciones de manera sistémica, no obstante y si bien es cierto que existen las disciplinas médicas y psicológicas, no existe una especialidad de los profesionales del área de la salud que los acerque de manera directa a la operación y al entorno aéreo, más bien éstos profesionales hacen parte de la organización como un segmento que opera de manera tímida ya que su inclusión carece del entrenamiento adecuado en materia técnica y cognitiva sobre la actividad aérea.

Si bien es cierto que, en materia del desarrollo y entrenamiento de las competencias para el personal aeronáutico, el profesional en psicología podría desempeñarse en actividades como: Diseño de sistemas y procesos, selección de personal, aptitud, entrenamiento, prevención y mucho más, estas actividades no se han incluido en la industria aeronáutica de manera holística y más bien se han direccionado (en la aviación no regular) casi con exclusividad hacia los centros de instrucción que ven el aporte del profesional como un requisito y, no como una estrategia de cambio, de crecimiento en competencias y competitividad; de otra parte y como ya se ha mencionado, éste profesional por 
su falta de competencia técnica aeronáutica participa como un eslabón del procedimiento más no como un elemento diferenciador y de gestión e inherencia en el ser.

\subsection{Organizaciones aeronáuticas}

Es claro que las organizaciones de aviación no regular y otros prestadores de servicios aéreos o de apoyo a la operación aérea, desarrollan procesos y procedimientos siempre y cuando éstos sean exigidos y vigilados por la autoridad ya que éste sector por su cultura organizacional no contiene en primera instancia procesos de certificación en calidad y no han sido permeadas por las competencias no técnicas, aún hoy en día los procesos de selección, habilitación y seguimiento del personal que labora en estas empresas, se basan en competencias técnicas y cognitivas más no en las actitudinales. En gran medida, las organizaciones de operación no regular están integradas por personal que no cumple los estándares de la aviación regular por no haber sido entrenados en los mismos, por ende la gestión de la seguridad operacional y la mitigación del riesgo operará como un componente específicamente técnico más no se obtendrá un cambio actitudinal y aun no se ve a corto plazo la inclusión de los programas y modelos diseñados a partir de los comportamientos del ser y aunque de manera global la accidentalidad ha disminuido, esto redunda indudablemente en las mediciones de la accidentalidad ubicando a la aviación no regular y de servicios especiales como el segmento que más aporta accidentes en la industria aeronáutica. (Figura 11)

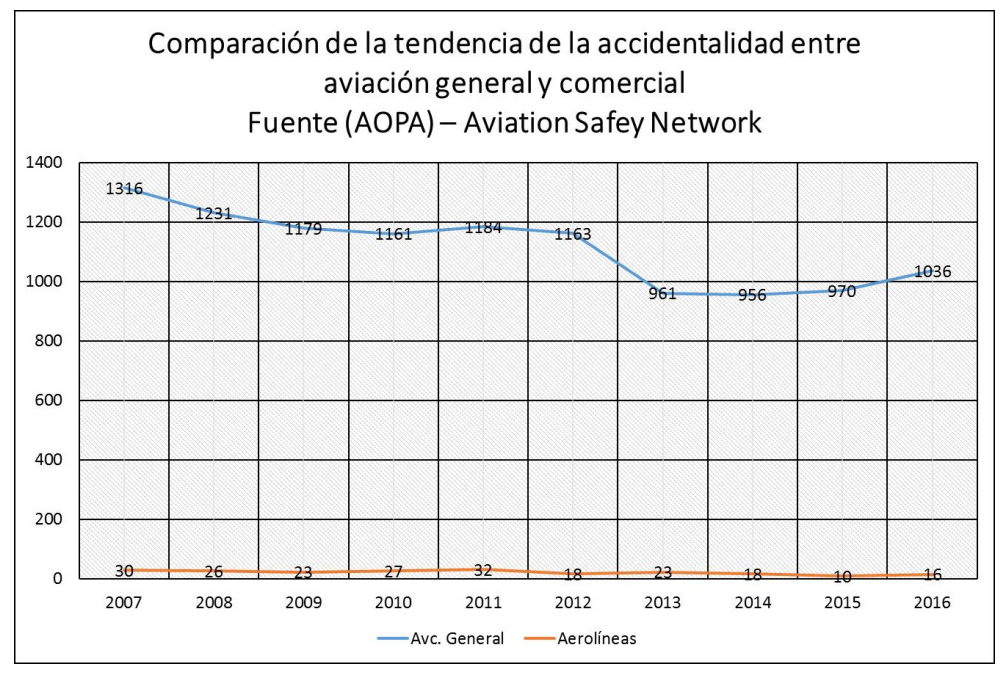

Figura 11: Accidentalidad de la aviación general por año (AOPA (Asociación de propietarios de avión y pilotos) - Aviation Safety Network)

\section{El principio de la integralidad}

El término integralidad no es nuevo, lo que tal vez sea novedoso es el papel que debe ocupar en los procesos de formación de personal y en la selección de personal basado en las habilidades no técnicas.

El concepto alude sobre todo a tres cuestiones que convergen obligatoriamente en el desempeño del personal, se trata de integrar en el sentido de articular pro eficiencias y habilidades específicas, 
esta triple integración no es fácil ya que implica cambios culturales profundos.

El principio de la integralidad se define como "La convergencia obligada que deben demostrar los individuos de la industria en tres aspectos claves del desempeño: Las habilidades técnicas, las habilidades cognitivas y las habilidades actitudinales". (Figura 12)

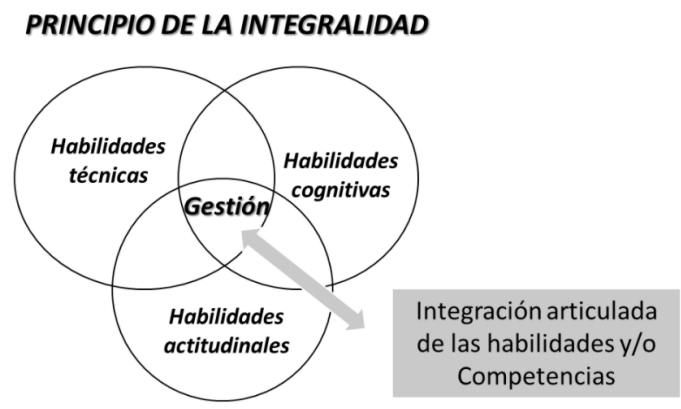

Figura 12: Principio de la Integralidad

El principio de la integralidad debe generar una transformación cultural dentro del sistema aeronáutico al incluir a las habilidades actitudinales como el elemento diferenciador en los individuos que son parte de la industria aeronáutica, esta transformación cultural está alineada con las características y objetivos de la seguridad operacional y el entrenamiento basado en evidencias y competencias o EBT (Evidence Based Training).

\subsection{El componente actitudinal}

El componente actitudinal hace referencia a las competencias y/o habilidades no técnicas que son sin lugar a duda el elemento primario de cambio frente a la capacidad de administrar el entorno operacional aeronáutico de manera sistémica y holística, este pilar ha de ser incluido como parte del perfil profesional de quienes pertenecen a la industria aeronáutica. Son ejemplos de habilidades actitudinales las siguientes: Trabajo en equipo, asertividad, toma de decisiones, mantenimiento de estándares, adherencia, planificación, gestión, administración, evaluación del riesgo, entre otros (Van Avermaete, 1998).

\subsection{Implementación del principio de la integralidad}

Como consecuencia de la necesidad de la industria en relación a la promoción de la cultura de la seguridad operacional, así como la necesidad de mantener los niveles del riesgo en los índices más bajos como sea razonable mediante un proceso continuo de identificación de peligros y gestión del riesgo, es indispensable que el principio de la integralidad se aplique en los procesos de entrenamiento y formación de personal, en la selección de personal aeronáutico incluyendo al personal administrativo de la industria, en las habilitaciones recurrentes y ascensos dentro de la organización así como en la promoción inter organizacional o modificación de los roles. 
Los modelos de gestión del riesgo (SHELL- C, TEM, LOFT, ALARP, CFIT, etc.) deberán entonces incluir el principio de la integralidad como una estrategia base que modifique el comportamiento de las personas en la toma de decisiones ante situaciones no deseadas.

La autoridad aeronáutica que tiene la responsabilidad de promover y vigilar el SMS de manera sistémica y el SSP como su propio sistema de gestión, se beneficia del principio de la integralidad en los programas de vigilancia de la industria y el aseguramiento de la calidad de sus propios procesos organizacionales actualizando con ello el criterio de las habilidades necesarias de la gestión, así mismo entrenando y seleccionando al personal bajo los lineamientos de este principio.

Las organizaciones de la industria que hacen parte del todo en el sistema aeronáutico y que buscan la asimilación de la cultura de la seguridad operacional, así como su certificación en calidad bajo los estándares del SMS, asegurarán la adherencia del personal en las buenas prácticas mediante el uso del principio de la integralidad como un factor elemental en el desempeño, ésta adherencia facilita la asimilación y el fortalecimiento de la cultura organizacional y con ello la de la seguridad operacional.

Los centros de instrucción que actualicen sus programas de formación incluyendo el componente actitudinal como un pilar diferenciador en la estructura del personal, mejoraran sin lugar a duda los estándares del perfil del egresado, así como el de sus instructores lo que redunda en un beneficio de competitividad ya que la inclusión del principio de la integralidad en el proceso de formación trae consigo la habilidad en la práctica y el uso de las defensas y herramientas especiales de gestión.

Finalmente, bajo el principio de la integralidad los profesionales del área médica en psicología tienen la oportunidad de desarrollar las habilidades técnicas y cognitivas necesarias para su desempeño y por otra parte trabajar en el reto del desarrollo de las habilidades no técnicas y/o actitudinales en el total de los individuos que hacen parte de la industria aeronáutica.

\section{Conclusiones}

Es preciso comprender a la industria aeronáutica de manera sistémica, esta industria es la interacción de conjuntos (organizaciones) que operan con un mismo fin, el fin no es otro que el de volar de un punto a otro de manera segura, éste ha sido sin lugar a duda el reto propuesto desde el inicio de la actividad aérea.

En el cumplimiento de este objetivo común, es de suma importancia el estudio permanente de la casuística y de las tendencias en materia de la mitigación del riesgo, hasta la fecha éste estudio ha arrojado resultados concluyentes en el hecho de que es "El Factor humano", el más representativo como determinante en la accidentalidad, hoy en día éste factor alude no solo al personal implicado directamente en la operación aérea, incluye además al personal técnico, administrativo, de control, autoridades y todos aquellos quienes intervienen en el conjunto de las organizaciones.

Dentro del estudio del factor humano se hace evidente que el comportamiento, es afectado de manera directa por los componentes fisiológico y psicológico de las personas; las personas deben ser entrenadas, evaluadas y promovidas dentro de los estándares que exige la industria; el reto es sin 
duda lograr el cambio actitudinal como una estrategia de crecimiento y de gestión, por lo tanto, en los perfiles administrativos, técnicos, financieros, de control, de la autoridad y operacionales deberá incluirse la habilidad actitudinal como un pilar en la formación de los individuos.

El principio de la integralidad que de manera articulada une a los factores y habilidades: técnicas, cognitivas y actitudinales, es el principio base para cualquier emprendimiento dentro del marco del cumplimiento de los objetivos trazados en materia de reducción de la probabilidad y la severidad de los eventos en la industria aeronáutica. 


\section{Referencias}

[1] Aviation Safety Network. https://aviation-safety.net/statistics/.

[2] Controlled Flight Into Terrain (CFIT). https://skybrary.aero/index.php/Controlled_ Flight_Into_Terrain_(CFIT).

[3] Flight Safety Foundation ALAR Toolkit. https://www. skybrary.aero/index.php/Flight_ Safety_Foundation_ALAR_Toolkit.

[4] NON-COMMERCIAL FIXED-WING GENERAL AVIATION ACCIDENT DATA: Non-Commercial Fixed-Wing. https://www.aopa.org/training-and-safety/ air-safety-institute/accident-analysis/joseph-t-nall-report/ non-commercial-fixed-wing.

[5] Threat and Error Management (TEM). https://www.skybrary.aero/index.php/Threat_ and_Error_Management_(TEM).

[6] VAN AVERMAETE. The Evaluation of Non-Technical Skills of Multi-Pilot Aircrew in Relation to the JAR-FCL Requirements. . Final Report NLR-CR-98443. Amsterdam: National Aerospace Laboratory (NLR), 1998.

[7] Barry G. Byrd. Slide Reference: slideshare, FAA course; failure to follow procedures, part 1. 2012.

[8] Organización de Aviación Civil Internacional. Anexo 19 - Sistema de la gestión de la seguridad operacional. Capítulo 4.

[9] Organización de Aviación Civil Internacional. Documento 9683 - Human Factors, Management and Organization. Human factor training manual, Chapter 2.

[10] Organización de Aviación Civil Internacional. Documento 9859 - Fundamentos de la gestión de la seguridad operacional. Manual de gestión de la seguridad operacional, Capítulo 2.

[11] ANDREW R. HALE and JAN JOVDEN. Ocupational injury, risk prevention and intervention, chapter eleven, management and culture, the third age of safety,a review of approaches to organizational aspects of safety, healt and enviroment. 1998.

[12] HERBERT WILLIAM HEINRICH. Industrial accident prevention a scientific. McGraw-Hill book company, inc, 1931.

[13] TE DEAL LG BOLMAN. Modern approaches to understanding and managing organizations. 1984.

[14] JAMES REASON. Human error: models and management. British Medical Journal 320 (7237): 768-770. PMC 1117770. PMID 10720363, Final Report NLR-CR-98443. Amsterdam: National Aerospace Laboratory (NLR), 2000.

[15] R WESTRUM. A typology of organisational cultures. Qual Saf Health Care 13(Suppl II):ii22-ii27, 2004. 